\title{
Effectiveness of an Awareness Programme on Drug Compliance among People with Selected Chronic Diseases
}

By Navaneetha.M

Abstract- The compliance to drug treatment leads to the prevention of deaths from the disease. Nurses play a very important role in the adherence to treatment by patients. The study aims at exploring the level of drug compliance among people with chronic illness (hypertension and diabetes mellitus), factors that influence the decision of the person and evaluates an awareness programme on the identified issues. The study used a Survey and Evaluative Approach with survey and quasiexperimental design. Purposive sampling was used to collect data from Marne, Athrady, Herebettu which are rural areas and Malpe area of Manipal which is an urban area. Among 23535 population surveyed a total of 1286 (602 urban and 684 rural) samples with the disease and undergoing treatment were identified. 328(184 urban and 144 rural) people who were not complying to drugs were given awareness programme. The tools used were Demographic performa, Morisky scale, Srivastava Socioeconomic scale, scale for Health status (SF-36) and a scale to assess factors. The study was based on the Rosentoch's, Becker and Maiman's Health Belief model.

Keywords: drug compliance, moriskyscale, srivastava socioeconomic scale, scale for health status (SF-36), health belief model, awareness programme.

GJMR-B Classification: NLMC Code: QV 55

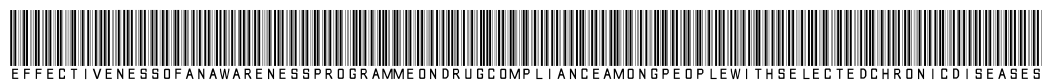

Strictly as per the compliance and regulations of:

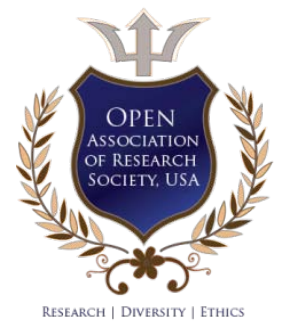

(c) 2021. Navaneetha.M. This is a research/review paper, distributed under the terms of the Creative Commons AttributionNoncommercial 3.0 Unported License http://creativecommons.org/licenses/by-nc/3.0/), permitting all non-commercial use, distribution, and reproduction in any medium, provided the original work is properly cited. 


\title{
Effectiveness of an Awareness Programme on Drug Compliance among People with Selected Chronic Diseases
}

\author{
Navaneetha. M
}

Abstract- The compliance to drug treatment leads to the prevention of deaths from the disease. Nurses play a very important role in the adherence to treatment by patients. The study aims at exploring the level of drug compliance among people with chronic illness (hypertension and diabetes mellitus), factors that influence the decision of the person and evaluates an awareness programme on the identified issues. The study used a Survey and Evaluative Approach with survey and quasiexperimental design. Purposive sampling was used to collect data from Marne, Athrady, Herebettu which are rural areas and Malpe area of Manipal which is an urban area. Among 23535 population surveyed a total of 1286 (602 urban and 684 rural) samples with the disease and undergoing treatment were identified. 328(184 urban and 144 rural) people who were not complying to drugs were given awareness programme. The tools used were Demographic performa, Morisky scale, Srivastava Socioeconomic scale, scale for Health status (SF-36) and a scale to assess factors. The study was based on the Rosentoch's, Becker and Maiman's Health Belief model. There was a significant association between area and level of drug compliance. $\left(\chi^{2}=19.087, p<0.001\right)$. Wilcoxin's sign rank test gave a significant relationship between pre and posttest knowledge scores. $(Z=-11.810$, $p=<0.001)$. There was also a significant difference between the posttest knowledge of experimental and control group $(Z=-7.540, p=<0.001)$. The study concludes that people with chronic diseases have drug compliance issues and there are factors influencing it ie knowledge and medical complexity and awareness programme will help to overcome it.

Keywords: drug compliance, moriskyscale, srivastava socioeconomic scale, scale for health status (SF-36), health belief model, awareness programme.

\section{INTRODUCTION}

$\mathrm{M}$ edication compliance is defined as the extent to which a patient takes the medication as prescribed. There are multiple studies in the literature that report non compliance rates of $30 \%$ to $50 \%$ or higher based on the class of agents and population studies, when medication was to be taken over a long period, compliance rates dropped dramatically to approximately $50 \%$ for either prevention or cure. The compliance to drug treatment leads to the prevention of deaths from the disease. In India studies of this nature are very few and hence the problem has to be explored.

Author: RNRM, PhD, Professor \& HOD, Dept of Community Health Nursing, College of Nursing, PIMS, Puducherry-14.

e-mail: navamd11176@gmail.com
A cross-sectional study was conducted by Cesar I. Fernandez-Lazaro etal, in primary healthcare centers of Spain which included 299 adult patients with $\geq 1$ chronic condition(s) and prescribed medication. The study used Morisky-Green-Levine questionnaire to assess medication adherence by interviews. 55.5\% were the proportion of adherent patients to treatment. The independent factors assessed were Older age, lower number of pharmacies used for medication refills $(0.65$, 95\% Cl 0.47-0.90), having received complete treatment information (3.89, 95\% Cl 2.09-7.21), having adequate knowledge about medication regimen $(4.17,95 \% \mathrm{Cl}$ 2.23-7.80), and self-perception of a good quality of life (2.17, 95\% Cl 1.18-4.02). To achieve appropriate levels of adherence tailored multifaceted interventions are required on the multidimensional factors found in this study, particularly those related to patients' education and their information needs.

The scope of the study developing a generic, individualized adhernce programme for chronic medication users was to describe the background for and content of an adherence counseling programme with a specific focus on an individualized, multidimensional adherence model for patients with a potential adherence problem (a so-called individualized systems model).

An intervention programme based on WHOs systems model for adherence was developed for implementation in primary health care and tested in a development project in Danish pharmacies in 2004-2005 by 27 patients in three pharmacies and 4 GP practices. Data were collected from the participants by registration forms, questionnaires, and focus groups. Since the programme was to support patients within the selfmanagement process regarding choice and implementation of medication treatment, various strategies were used and different theoretical assumptions and choices made before fixing the study. The strategies used include differentiating the differing kinds of non-adherence, a model for stages of change, self-efficacy, narratives, motivating interviewing strategies and training techniques. The strategies and theoretical reflections led to the formation of a counselling programme, which was tested in two forms, a basic and an extended version - provided by either a pharmaconomist or a pharmacist. Besides, the results 
include a description of how the WHO-model is transformed into an individualized counseling model. According to WHO, non-adherence should not be viewed as an isolated, single-factor problem, but rather as a multi-dimensional problem not determined exclusively by patient factors, as is seen most often in adherence research. WHOs systems model aims to analyze and provide explanations for non-adherence on a societal and health policy level in a broader sense.

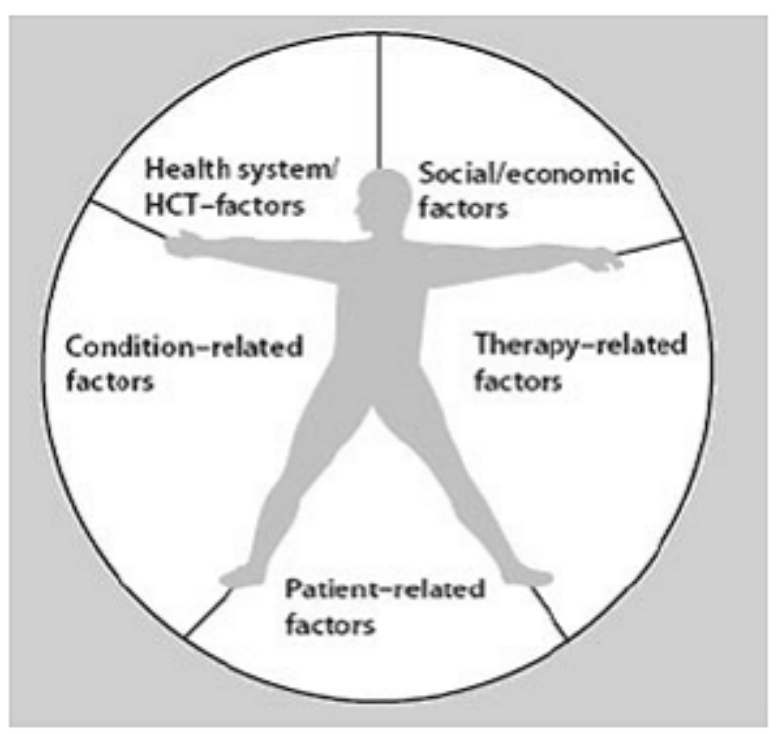

Figure 1: The five dimensions of adherence as suggested by the World Health Organisation

The programme identifies potential nonadherence, analyses the character of the issues identified, such as drug-related problems, explores patient resources and provides concordance-based follow-up sessions and individually based interventions.
The model developed and used as a template for the entire programme was called the individualized systems model. It emerged from the transformation of the WHO model into an individualized counseling model.

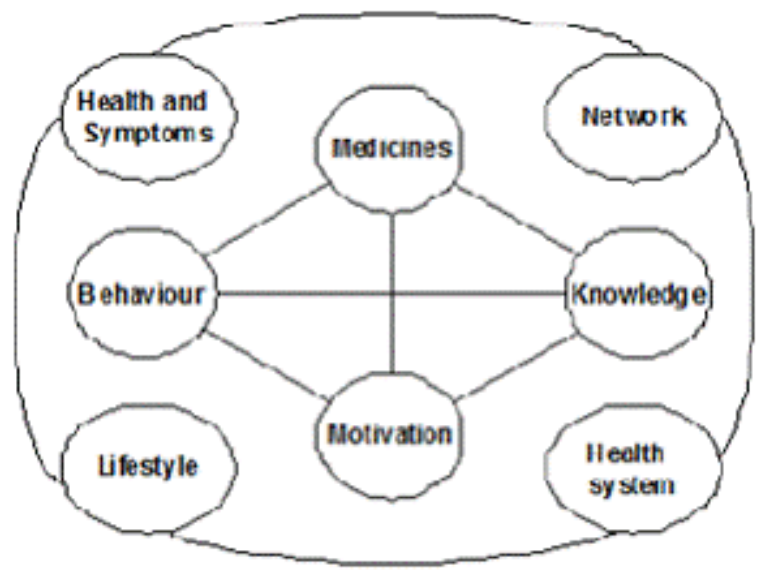

Figure 2: The individualised systems model

Usha Malagi, Rama Naik and Ramesh Babruwadin their study on Knowledge Practices and Life Style Factors of Type - 2 Diabetics has found that the life style factors such as foods restricted and specially included, vices prevalent, exercise behavior and knowledge and practices of 50 type-2 diabetics were assessed using a pretested structured questionnaire. Diabetics restricted the foods such as rice, roots and tubers, sweets and fruits. The foods were specially included for the management of disorder by majority of diabetics (72\%). The food which was specially included was green leafy vegetables, bitter gourd, salads, ragi and spices. The habits practiced by men were smoking (14\%), drinking alcohol (48\%) and tobacco chewing was seen in very few men and women. Exercise was done by half of the diabetics (56\%) and half of the exercising subjects had started exercise only about a year back. About 30 and 16\% diabetics had poor knowledge of diabetes practices. Thus, the diabetics need education to improve the knowledge and practices for the proper management of disorder. 
The EAPACUM-HTA study in Spain at 40 primary care centres conducted for 6 months with newly diagnosed or uncontrolled hypertension included 250 patients. They were given an electronic monitor for measuring compliance (monitoring events medication system).Compliance observed was $74 \%$ and $92 \%$ in control group and intervention group.(95\% Cl 81.2-94 and $80.7-98.3 ; P=0.0001$ ). The number need to treat to avoid one case of noncompliance was 5.6 patients. The programme was found effective in improving compliance in arterial hypertension.

Nurses play a very important role in the adherence to treatment by patients. In the paper Nursing Care Management and Responsibility it is stated that: Improving patient compliance with treatment orders through health education and extending care to all patients, education will help patients to improve drug compliance.

The study aims at exploring the factors that influence the decision of the person suffering from chronic diseases, to comply with the regular drug regimen prescribed for them. The study also recommends means to organize an awareness programme on the identified issues. Thus ultimately contributing to one of the goal set by WHO i.e. to reduce death rates in chronic diseases.

The objectives of the study are to:

1. Assess the level of drug compliance and identify the factors influencing it

2. Plan and evaluate the effect of an awareness programme on drug compliance

\section{Methodolgy}

Research Approach: Survey and Evaluative Approach

\begin{tabular}{|c|l|l|l|}
\hline Phases & Design & \multicolumn{1}{|c|}{ Instrument } & \multicolumn{1}{|c|}{ Plan of data analysis } \\
\hline I & Survey & $\begin{array}{l}\text { Demographic variables } \\
\text { Moriski scale-scale to assess level of } \\
\text { drug compliance }\end{array}$ & $\begin{array}{l}\text { Percentage, Prevalence } \\
\text { rate, mean, chi-square }\end{array}$ \\
\cline { 3 - 5 } & & $\begin{array}{l}\text { Scale on Health status(SF-36) } \\
\text { Scale on complexity of medication, } \\
\text { patient knowledge, social support and } \\
\text { patient provider interaction }\end{array}$ & $\begin{array}{l}\text { Multiple logistic regression, } \\
\text { other tests }\end{array}$ \\
\hline II & Quasi experimental & Morisky scale and scale on knowledge & Non-parametric tests \\
\hline
\end{tabular}

Setting and Population: The target population of the study comprised of the people with the disease conditions and undergoing treatment in the selected rural and urban areas ie Marne, Athrady, Herebettu which are rural areas and Malpe area of Manipal which is an urban area.

a) Sample and Sampling technique

Purposive sampling was used

\begin{tabular}{|c|c|}
\hline Area & Population \\
\hline Rural & \\
Kidiyoor-Malpe & 4546 \\
Herebettu & 2302 \\
Marne & 2734 \\
Alevoor & 2101 \\
Total & 11683 \\
\hline Urban & 5152 \\
Kalmady-Malpe & 6700 \\
Kodavoor A-Malpe & 11852 \\
\hline Total & \\
\hline
\end{tabular}

Among this a total of 1286 (602 urban and 684 rural) samples with the disease and undergoing treatment were identified. 328(184 urban and 144 rural) people who were not complying to drugs were given awareness programme.

\section{b) Procedure for Data Collection}

House to house survey was done and people with either of the diseases taking any system of medicine were given the questionnaire. Those identified with drug non compliance was given teaching and there level of compliance and knowledge was assessed after 15 days.

Data analysis was done based on the objectives and hypotheses stated in the study by using descriptive and inferential statistics

c) Description of tool

The following tools were used Demographic performa, Morisky scale, Srivastava Socioeconomic scale, scale for Health status (SF-36) and a scale to assess factors. 
The Demographic performa consisted of variables including age, sex, education, occupation, place of residence, socio economic status, nature of disease and nature of treatment.

Morisky scale was used to assess the level of drug compliance which is a self administered tool. It includes 4 statements with Yes/no. It is measured as 0sure high adherence, 1-2- Medium adherence, 3-4- low adherence.

The Srivastava Socioeconomic scale was used in the study.

The factors were assessed with scales for Health status (SF-36) which is a scale with 36 questions to assess a person's health status and one prepared with statements on knowledge of patient, medical complexity, social support and patient-provider interaction. Each statement consists of 3 optionsalways, sometimes, never. This tool was purchased from author.

The content validity index was 0.86 .

The reliability of the tools was found to be $\alpha=0.8231$ by Cronbach alpha method.

\section{ili. Conceptual Framework}

The study was based on the Rosentoch's, Becker and Maiman's Health Belief model. This model was developed to provide a framework for understanding why some people take specific actions to avoid illness, whereas others fail to protect themselves. The model was designed to predict which people would and would not use preventive measures and suggest interventions that might reduce client's reluctance to assess health care. There are three major components of the health belief model: individual perceptions, modifying factors and likelihood of action. In addition uses of cues to action such as mass media campaigns, advice from others, illness of family members or friends and newspaper and magazine article may help to motivate clients to take action.

The health belief model is beneficial in assessing health protection or disease prevention behaviours. It is also useful in organizing information about clients' views of their state of health and what factors may influence them to change their behavior. The model, when used appropriately provides organized assessment data about client's abilities and motivation to change their health status. Health education programs can be developed to fit the clients.

In this study the first component was individual perception which is the non compliance to drugs of hypertension and diabetes mellitus.

The second component was modifying factors which include the demographic variables and structural variables. The demographic variables include age, sex, socioeconomic status and nature of treatment. The structural variables are the factors which will influence the drug compliance.
The third component is likelihood of action, which includes perceived benefits minus perceived barriers for preventive action. In this study the benefit will be the gain in knowledge by the client which will lead to change in behavior. The barriers may be the number of drugs taken and socioeconomic status, etc. In addition to this the components on cues to action is the health awareness programme which can motivate the client to take action. 
Individual

Perceptions
Modifying Factors

Likelihood of

Action

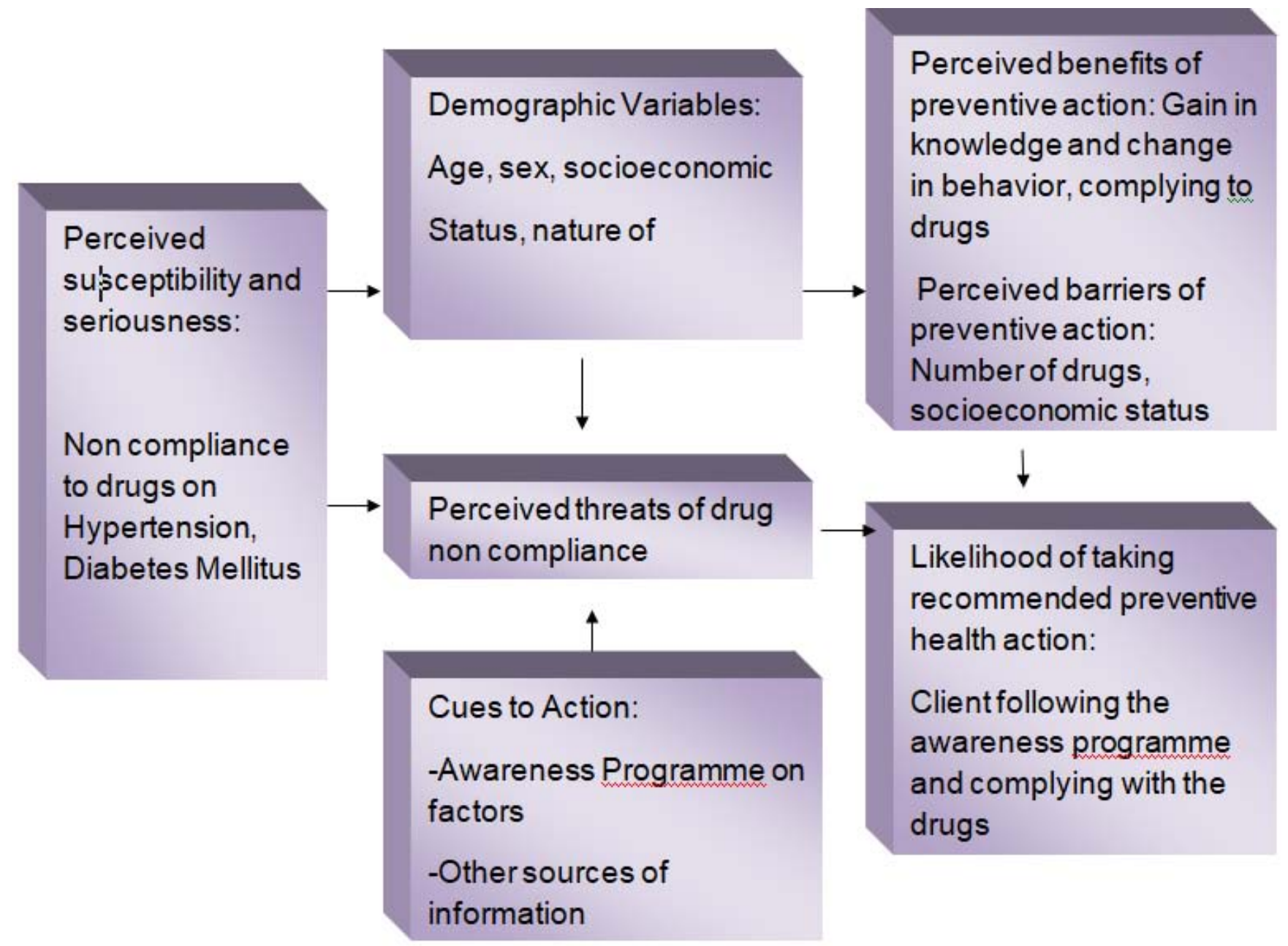

Figure 3: Conceptual Framework based on Rosenstoch's and Becker and Maiman's Health Belief Model

\section{Results}

Table-1: Frequency and percentage distribution of demographic characteristics

$n=1286$

\begin{tabular}{ccccc}
\hline \multirow{2}{*}{$\begin{array}{c}\text { Sample } \\
\text { characteristics }\end{array}$} & \multicolumn{4}{c}{ Area } \\
\cline { 2 - 5 } & \multicolumn{2}{c}{ Rural $(\mathrm{n}=684)$} & \multicolumn{2}{c}{ Urban $(\mathrm{n}=602)$} \\
\cline { 2 - 5 } Age & 53 & 7.75 & 128 & Prequency \\
Percentage & 182 & 3.26 \\
$30-40$ & 246 & 35.96 & 174 & 28.90 \\
$41-50$ & 292 & 42.69 & 118 & 19.60 \\
$51-60$ & 93 & 13.60 & & 47.18 \\
61 and above & & & 284 & 52.82 \\
Sex & 296 & 43.27 & 318 & \\
Male & 388 & 56.73 & & \\
Female & & & & 40.86 \\
Socio & & & 246 & 51.83 \\
Economic & & 60.38 & 312 & 7.31 \\
status & 413 & 38.74 & 44 & \\
Low & 265 & 0.88 & & \\
Medium & 6 & & & \\
High & & &
\end{tabular}


The data represented in Table- 1 show that, out of 1286 subjects, 684 (53.19\%) belonged to rural area and 602 (46.81\%) belonged to urban area. 246 (42.69\%) belonged to 51 - 60 years of age in rural area and in urban area $182(30.23 \%)$ belonged to $41-50$ years. In both areas most of the samples were females i.e. 388 (56.73\%) and 318 (52.82\%) respectively. In rural
413(60.38\%) belonged to the low socioeconomic status and in urban $312(51.83 \%)$ belonged to the medium category.

The sample was classified as low, medium and sure adherence to medication based on the details of Morisky scale.

$$
\text { Table -2: Level of drug compliance } \quad \mathrm{n}=1286
$$

\begin{tabular}{ccccc}
\hline Level of Drug Compliance & \multicolumn{4}{c}{ Area } \\
\cline { 2 - 5 } & \multicolumn{2}{c}{ Rural $(\mathrm{n}=684)$} & \multicolumn{2}{c}{ Urban $(\mathrm{n}=602)$} \\
\cline { 2 - 5 } & Frequency & Percentage & Frequency & Percentage \\
\hline Sure Adherence & 540 & 78.95 & 418 & 69.44 \\
Medium Adherence & 90 & 13.16 & 95 & 15.78 \\
Low Adherence & 54 & 7.89 & 89 & 14.78 \\
\hline
\end{tabular}

The data in Table-2 describes the sample in terms of their level of drug compliance. In rural area $540(78.95 \%)$ were adhering to drugs were as in urban area only $418(69.44 \%)$ were adhering to the drugs.

There was a significant association between area and level of drug compliance. $\quad\left(\chi^{2}=19.087\right.$, $p<0.001)$

Among the factors identified that is Knowledge, Medical complexity, Social relations (Husband/wife, Family member, Friends) and Patient provider interaction system of medicine, medication prescribed. , SF 36 only factors of knowledge $\left(\chi^{2}=113.081\right.$, $\mathrm{p}<0.001)$, medical complexity $\left(\chi^{2}=90.814, p<0.001\right)$ and relation of husband or wife $\left(\chi^{2}=7.831, p=0.02\right)$ were significant. It was also found that there was a significant relationship between the area and factors for knowledge of medicines taken $(Z=-2.708, p=0.007)$, relationship between sample and family member and friends to motivate to take medicines $(Z=-4.668$, $p<0.001, Z=-4.527, p<0.001)$ and the health status score SF-36( $Z=-2.117, p=0.034)$.

Further a regression analysis was done with the factors associated and it is concluded that there is a relationship between knowledge $(\mathrm{OR}=1.28, \mathrm{Cl}-1.20$ $1.35, \mathrm{p}<0.001)$, medical complexity $(\mathrm{OR}=1.14, \mathrm{Cl}-$ 1.10-1.19, $p<0.001$ ) and the people getting drug metformin $(\mathrm{OR}=0.278, \mathrm{Cl}-0.08-0.88, \mathrm{p}<0.03)$ with sure complying of drugs. In medium compliance there was a relation with hypertension $(\mathrm{OR}=2.70, \mathrm{Cl}-1.39-5.24$, $\mathrm{p}=0.003)$, diabetes $(\mathrm{OR}=2.84, \mathrm{Cl}-1.42-5.68, \mathrm{p}=0.003)$, knowledge $(\mathrm{OR}=1.14, \mathrm{Cl}-1.07-1.22, \quad \mathrm{p}<0.001)$ and medical complexity $(\mathrm{OR}=1.05, \mathrm{Cl}-1.01-1.10, \mathrm{p}=0.16)$. Hence it is concluded that knowledge and medical complexity were the two factors affecting drug compliance.

Table -3: Association between level of drug compliance among experimental and control groups in post test $n=328$

\begin{tabular}{|c|c|c|c|c|c|c|}
\hline \multirow{2}{*}{ Group } & \multicolumn{3}{|c|}{ Level of drug compliance } & \multirow{2}{*}{$\chi^{2}$} & \multirow{2}{*}{ df } & \multirow{2}{*}{$\mathrm{p}$} \\
\hline & sure & medium & low & & & \\
\hline Experimental & 155 & 7 & 2 & \multirow{2}{*}{282.14} & \multirow{2}{*}{2} & \multirow{2}{*}{$<0.001$} \\
\hline Control & 3 & 121 & 40 & & & \\
\hline
\end{tabular}

Table -4: Association between level of drug compliance in pre and post test in Experimental group $\mathrm{n}=164$

\begin{tabular}{c|c|c|c|c|c|c}
\hline \multirow{2}{*}{ Pretest } & \multicolumn{3}{|c|}{ Level of drug compliance } & \multirow{2}{*}{$\chi^{2}$} & \multirow{2}{*}{ df } & \multirow{2}{*}{$p$} \\
\cline { 2 - 4 } & Sosttest & Medium & Low & & & \\
\hline Medium & 92 & 91 & 2 & \multirow{2}{*}{57.00} & 2 & $<0.001$ \\
\hline Low & 66 & 37 & 40 & 2 & \\
\hline
\end{tabular}

Both tables show that there is a significant difference between level of drug compliance after the teaching and counseling programme. Further the knowledge aspect which was relating to the need for medicine intake was also assessed in pre and posttest of experimental group, Wilcoxin's sign rank test gave a significant relationship. $(Z=-11.810, p=<0.001)$.

There was also a significant difference between the posttest knowledge of experimental and control group $(Z=-7.540, p=<0.001)$ 
Table -5: Relation between level of drug compliance and knowledge in posttest $(\mathrm{N}=328)$

\begin{tabular}{c|c|c|c|c|c|c}
\hline & \multicolumn{3}{|c|}{ Level of drug compliance } & \multirow{2}{*}{$\chi^{2}$} & df & \multirow{2}{*}{$p$} \\
\cline { 2 - 4 } & Sure & Medium & Low & & & \\
\hline Post test knowledge & 158 & 128 & 42 & 66.728 & 2 & $<0.001$ \\
\hline
\end{tabular}

There was a relationship between the knowledge and level of drug compliance in posttest of experimental group $\left(\chi^{2}=66.728, p<0.001\right)$.

With all the above it is concluded there was a difference in the level of compliance between pretest and posttest

\section{Discussion}

The study on Self-Reported Morisky Score for Identifying Nonadherence with Cardiovascular Medications reports that the Morisky medication adherence scale is a commonly used adherence screening tool. It is composed of 4 yes/no questions on past medication use patterns. Forty-nine of 377 (13\%) patients were categorized as non adherent; however, only $12(3 \%)$ patients had Morisky scores suggesting a high likelihood of non adherence (3 or 4). The present study has identified 114(13.64\%) medium and 98(11.72\%) low out of 836 hypertensive patients.

In a study conducted by glycemic control and medication compliance in diabetic patients in a pharmacist managed clinic in Hong Kong; non compliant patients were assessed by nurses and sent to the pharmacist. The clients had to visit the clinic three times. Out of 95 patients, 91 gave complete data. The compliance rate at the beginning and at the end of third visit was $41.3 \pm 25.6$ and $97.8 \pm 1.6, p<0.005$. In the current study the diabetes with level of compliance and low were 116. The reasons for non compliance stated in the study is similar to the study findings with Forgetfulness $61.5 \%$, Adverse effects $25.3 \%$, Wrong belief about treatment $8.8 \%$, Not realizing that the treatment had been changed $6.6 \%$ and others $2.2 \%$ which are the points assessed under knowledge and medication complexity. These concepts had a scale score of mean $14.55 \pm 3.541$ standard deviation in rural and $15.27 \pm 4.323$ in the urban area and for medication complexity20.31 \pm 5.859 in rural and $20 \pm 6.433$ in urban.

Another supporting study related to negative medication belief conducted in Kuwait as a prospective cross-sectional study for patients treated for chronic illnesses in the Ministry of Health primary care clinics was to identify self-reported adherence to medications, beliefs, and perceived sensitivity toward medications. Of the response rate of $68.1 \% .56 .7 \%$ were male, $73.7 \%$ were married, $53.3 \%$ were non-Kuwaitis, and $49.4 \%$ had low income and had a mean of two comorbid illnesses. They had indicated taking an average of four prescription medicines to treat them. A structural equation model analysis showed that the following are negatively impacting adherence to medications: higher negative beliefs toward medications (beta $=-0.46$ ), marital status (being unmarried; beta $=-0.14$ ), nationality (being Kuwaiti; beta $=0.15$ ), lower education level (beta $=-0.14)$, and higher illness severity (beta = 0.15 .

A study on Compliance and knowledge of hypertensive patients attending $\mathrm{PHC}$ centres in $\mathrm{AL}$ Khobar, Saudi Arabia was a cross-sectional study of all hypertensive patients (190) attending four primary health centres. The mean age was $49.9 \pm 11.7$ years, the overall compliance rate was $34.2 \%$ which was lower in those aged $<55$ years than older patients $(26.2 \%$ versus $48.5 \%, p<0.001)$ and among educated than illiterate $(30.4 \%$ and $38.1 \%$ respectively, $\quad p<0.001)$. The knowledge level regarding the disease was very minimal as $41.6 \%$ of the patients thought that hypertension could have a permanent cure and $43.7 \%$ that medication could be stopped once control was achieved. As to the etiology $66.3 \%$ thought as emotional stress and $1.6 \%$ as heredity. Hence an education on hypertension is essential among these patients. Among the people who did not comply to medicine most of them were hypertensives ie a total of 160 and most of them where in the age group of 51-60 years in both rural and urban area. In rural the level of compliance was 63(43.75\%) and urban 56(30.43\%). The females did not comply to drugs in both groups and they belonged to medium socioeconomic status.

In the study the multilevel compliance challenge; it is stated that compliance is a complex behavioural pattern strongly influenced by the environments in which the patients live, healthcare providers practice and health care systems delivery of care. The health care providers including pharmacists, nurses, psychologists etc who are involved in primary and secondary prevention play a role in enhancing compliance by interpreting recommendations, educating and motivating patients, monitoring responses to recommended behaviours and providing feedback. Maximum use of these services should be made by patients to overcome non compliance to drugs. Multilevel approach of education and behaviour change is important like consumer health education, provider education, etc.

In a study on assessment of impact of medication counseling on patients' medication knowledge and compliance in an outpatient clinic in South India explains that there is an improvement in the compliance among the group of patients who were counseled against the usual care group. (92.29 \pm 4.5 and $84.71 \pm 11.8 \%)$ Knowledge level of the counseled group also showed an improvement (13.82 \pm 1.8604 and 
$11.78 \pm 3.5037)$. The current study also shows a significant difference in patient's level of compliance after an awareness programme $\left(\chi^{2}=282.14, p<0.001\right)$, the study also reveals a significant difference in knowledge levels $(Z=-7.540, p<0.001)$.

\section{Vi. Conclusion}

The study concludes that medication compliance differs in urban and rural populations, reasons mainly being knowledge and medical complexity. It also found appropriate awareness programme conducted can bring a change in the compliance.

\section{References Références Referencias}

1. Adherence to long term therapies-Evidence for Action WHO. 2003;18-20

2. Burnier M. Compliance in hypertension. EDTNA ERCA J.2005; 31(3):152-155

3. Miller NH etal. The Multilevel Compliance Challenge: Recommendations for a Call to action A statement for health care professionals. American Heart Association. 1997;95:1085-1090

4. Nursing care Management and responsibilities: Improving patient compliance with treatment orders through health education and extending care to all patients. Available from: www.lotsofessays.com/ viewpaper/1687355.html

5. L.S.Al-Sowielem, A.G.Elzubier. Compliance and knowledge of hypertensive patients attending PHC centres in Al-Khobar, Saudi Arabia. Eastern Mediterranean Health Journal. 1998; 4(2):301-307

6. Lee $W$ Y, Leung PK. Glycemic control and medication compliance in diabetic patients in a pharmacist managed clinic in Hong Kong. Am J Health-Syst Pharm. 2003; 60: 2593-2596

7. Shalansky S J, Levy A R, Ignaszewski A P. SelfReported Morisky Score for Identifying Nonadherence with Cardiovascular Medications. The Annals of Pharmacotherapy. 2004; 38(9): 1363-1368.

8. Ponnusankar $\mathrm{S}$ etal. Assessment of impact of medication counseling on patients' medication knowledge and compliance in an outpatient clinic in South India. Patient Educ Couns. 2004; 54(1): 55-60

9. Hanne Herborg, Lotte S, Charlotte Rossing, Pernille DAM. Developing a generic, individualised adherence programme for chronic medication users. Pharmacy Practice (Internet). 2008; 6(3) Available from Journal@pharmacypractice.org doi: 10.4321/S1886-36552008000300006

10. Usha Malagi, Rama Naik Ramesh Babruwad. Knowledge Practices and Life Style Factors of Type - 2 Diabetic. Karnataka J. Agric. Sci.2007; 20(4): 823-826

11. Marquez-Contreras E etal. Efficacy of a home blood pressure monitoring programme on therapeutic compliance in hypertension: the EAPACUM-HTA study. J Hypertens. 2006; 24(1): 169-175

12. Srivastava. Socioeconomic scale. Delhi: Manasayan.1986

13. Quality Metric Org www.sf-36.org/tools/sf36.html

14. Potter PA. Perry AG. Basic nursing essentials for practice. $6^{\text {th }}$ ed. Missouri: Mosby, 2007;p. 4,5

15. Cesar I. Fernandez-Lazaro, et al. (2019) 'Adherence to treatment and related factors among patients with chronic conditions in primary care: a cross-sectional study' BMC Family Practice volume 20, Article number: 132

https://bmcfampract.biomedcentral.com/articles/10. 1186/s12875-019-1019-3

16. Lemay J, Waheedi M, Al-Sharqawi S, Bayoud T 'Medication adherence in chronic illness: do beliefs about medications play a role?' Dovepress, Volume 2018:12 Pages 1687-1698

https://www.dovepress.com/medication-adherencein-chronic-illness-do-beliefs-about-medications-ppeer-reviewed-fulltext-article-PPA 